\title{
Paramedics Manage the Airway Easier with Laryngeal Mask Airway Than with Intubation during Simulated CPR Scenarios
}

\author{
Eleni Bassiakou, Konstantinos Stroumpoulis, and Theodoros Xanthos \\ Department of Anatomy, School of Medicine, University of Athens, 11527 Athens, Greece \\ Correspondence should be addressed to Eleni Bassiakou, nbassiakou@yahoo.com
}

Received 31 May 2012; Accepted 11 July 2012

Academic Editors: P. Eisenburger and O. Karcioglu

Copyright ( $) 2012$ Eleni Bassiakou et al. This is an open access article distributed under the Creative Commons Attribution License, which permits unrestricted use, distribution, and reproduction in any medium, provided the original work is properly cited.

Objectives. In this study, we investigated whether minimally trained paramedics with no previous experience in securing the airway would be more efficient in placing laryngeal mask airway (LMA) than intubating the trachea with direct laryngoscopy using 2 manikin scenarios: without chest compressions (non-CPR scenario) and with continuous chest compressions (CPR scenario). Methods. One hundred and twenty-four paramedics with no previous experience in managing the airway comprised our study population. Three $20 \mathrm{~min}$ lectures on the anatomy of the airway and endotracheal intubation with the Macintosh blade and LMA placement were given. Each participant attempted to insert a LMA or to intubate a manikin with the Macintosh blade using 2 different scenarios: without chest compressions and with continuous chest compressions. Results. All participants managed to place a LMA in both scenarios, but only $85 \%$ of them managed to intubate the trachea using the Macintosh blade in the non-CPR and even less than $(80 \%)$ in the CPR scenario. Insertion of the LMA was statistically significantly faster than intubation with the Macintosh blade in both scenarios $(P<0.001)$. Conclusions. Our results provide evidence that for minimally trained paramedics, LMA insertion is much faster than endotracheal intubation.

\section{Introduction}

Management of the airway is of utmost importance in the cardiopulmonary resuscitation (CPR) setting. Different devices and techniques, such as the endotracheal intubation and the laryngeal mask airway (LMA), have been recommended. Although standard direct laryngoscopic tracheal intubation remains the preferred technique to establish advanced airway management, sufficient experience is required to gain proficiency, and constant retraining is required in order to maintain the skill [1]. The classic laryngeal mask airway (LMA) on the other hand is easy to insert and when compared to endotracheal intubation is easier to learn $[2,3]$.

In the prehospital setting, airway management is often performed by paramedics under suboptimal conditions. Paramedic intubation has been a widely practiced aspect of prehospital cardiac arrest care for the past 20 years [4, 5]. However, survival after prehospital cardiac arrest may be improved by reducing paramedic intubation time [6].
This intrigued us to investigate whether minimally trained paramedics with no previous experience in securing the airway would be more efficient in placing LMA or in intubating the trachea with direct laryngoscopy in a manikin study. Due to the fact that the latest European Resuscitation Council (ERC) Guidelines emphasize minimizing interruptions to chest compressions, in order to maximize coronary and cerebral perfusion pressure, the study was designed using 2 manikin scenarios: non-CPR and CPR with uninterrupted chest compressions $[7,8]$. The primary endpoints in this study were success rate and time for LMA insertion or endotracheal intubation in these 2 scenarios.

\section{Materials and Methods}

Paramedic personnel graduating in June 2010 were contacted. In total, 213 paramedics were identified and out of these, 152 consented to participate in the present study. After excluding those with previous experience in managing the 
airway with any technique, 124 paramedics comprised our study population. All were informed about the purpose of the study, and they were asked to sign an informed consent. Institutional Ethics Committee approval was not required as this was a manikin study with volunteers, and it did not involve humans or animals.

In order to train the participants, the investigators gave three 20 min lectures on the anatomy of the airway and endotracheal intubation with the Macintosh blade and LMA placement. The equipment was demonstrated in detail to the participants, who were then allowed to familiarize themselves with it. Using sealed envelopes, participants were allocated to 2 groups in order to practice each technique. Randomization was achieved with 124 integers, one for each participant, as previously described [9]. The 2 groups were trained in the 2 techniques using a crossover design. Each participant attempted to insert a LMA or to intubate a manikin with the Macintosh blade until one successful attempt was recorded. If the participants failed to do so after 5 attempts, this was recorded as a failure. Each participant attempted intubation using 2 different scenarios: without chest compressions and with continuous chest compressions. Participants were not allowed to watch each other during any of the intubation attempts with each device, in order to avoid any learning effects throughout the procedure.

A size 4 LMA (The Laryngeal Mask Company Limited, Buckinghamshire, UK) was used for the purpose of the study. All intubations were performed using a size of $7.5 \mathrm{~mm}$ endotracheal tube (ETT) (Mallinckrodt Medical, Athlone, Ireland) and a size 3 Macintosh blade. An adult Resusci Anne manikin with an Airway Trainer head (Laerdal, Stavanger, Norway) was used placed in the "sniffing position" in a standard hospital bed. Both the ETT/LMA and the manikin were appropriately lubricated according to the manufacturer's instructions. Chest compressions were performed with a mechanical chest compressor (LUCAS Chest Compression System, Jolife AB, Mantzaris, Greece). LUCAS provides the same quality of chest compressions independent of rescuer fatigue, or variability in the experience level of the caregiver.

After the educational session, the participants were assessed in their ability to place a LMA and to intubate the trachea. The time required for the first successful attempt was defined as the time from insertion of the blade or the LMA between the teeth until the ETT or the LMA was deemed to be correctly placed, and its cuff was properly inflated by each participant [9]. A single observer using always the same stopwatch recorded the time elapsed and confirmed correct ETT/LMA placement. Effective ventilation of the manikin with a self-inflating bag was considered to be a successful attempt. Failure to intubate the trachea and time to ETT/LMA insertion longer than 30 seconds were defined as unsuccessful attempts. Failure to ventilate both lungs with the LMA when using a self-inflating bag was also considered to be a failed attempt. The primary endpoints were the number of attempts and the time required for the first successful attempt of tracheal intubation or LMA placement. Secondary endpoint was the participants' level of self-confidence regarding the use of each technique. The participants were asked to comment on their level of
TABLE 1: Number of attempts for successful securing of the airway with each technique; CPR: cardiopulmonary resuscitation.

\begin{tabular}{lcccc}
\hline \multirow{2}{*}{ Number of attempts } & \multicolumn{2}{c}{ LMA } & \multicolumn{3}{c}{ Macintosh blade } \\
& Non-CPR & CPR & Non-CPR & CPR \\
\hline 1st & 96 & 92 & 61 & 30 \\
2nd & 18 & 24 & 31 & 45 \\
3rd & 8 & 8 & 8 & 14 \\
4th & 2 & 0 & 5 & 10 \\
Failed attempts & 0 & 0 & 19 & 27 \\
\hline
\end{tabular}

LMA: $P=0.2$ in the non-CPR versus the CPR scenario. Macintosh blade: $P=0.003$ in the non-CPR versus the CPR scenario. LMA versus Macintosh blade (non-CPR scenario): $P<0.001$. LMA versus Macintosh blade (CPR scenario): $P<0.001$.

self-confidence, concerning the new skills. The confidence was evaluated using a $0-10$ scale $(0=$ no confidence, $10=$ absolute confidence) .

2.1. Statistical Analysis. As data were not normally distributed, nonparametric tests were applied. We used the median test for continuous variables and the uncertainty coefficient test for categorical data. Results are presented as median values with range. $P$ value $<0.05$ was considered to be statistical significant. The SPSS statistical package (SPSS, Chicago, IL, Version 17 for Windows) was used for all analyses.

\section{Results}

One hundred and twenty-four paramedics were included in the present study. All participants managed to place a LMA in both scenarios, but only $85 \%$ (105 out of 124) of them managed to intubate the trachea using the Macintosh blade in the non-CPR and even less $(80 \%, 99$ out of 124) in the CPR scenario.

The median time required for the first successful placement of LMA was $12(8-22) \mathrm{sec}$ in the non-CPR scenario and $12(6-22) \mathrm{sec}$ in the CRP scenario $(P=1)$ while intubation with the Macintosh blade was 15 (9-28) sec in the non-CPR scenario and 19 (10-29) sec in the CPR scenario $(P=0.002)$. Insertion of LMA was statistically significantly faster than intubation with the Macintosh blade in both scenarios $(P<0.001)$ (Figure 1). Furthermore, no statistically significant difference was observed in the number of attempts required for the first successful LMA insertion in the non-CPR scenario versus the CPR scenario $(P=0.2)$, whereas in intubation with the Macintosh blade statistical significance was observed in the non-CPR versus the CPR scenario $(P=0.03)$. Moreover, the number of attempts for the first successful airway management was significantly lower for the LMA in both scenarios (Table 1).

Finally, participants expressed a greater level of selfconfidence in using the LMA instead of intubating the trachea with the Macintosh blade (Table 2). 


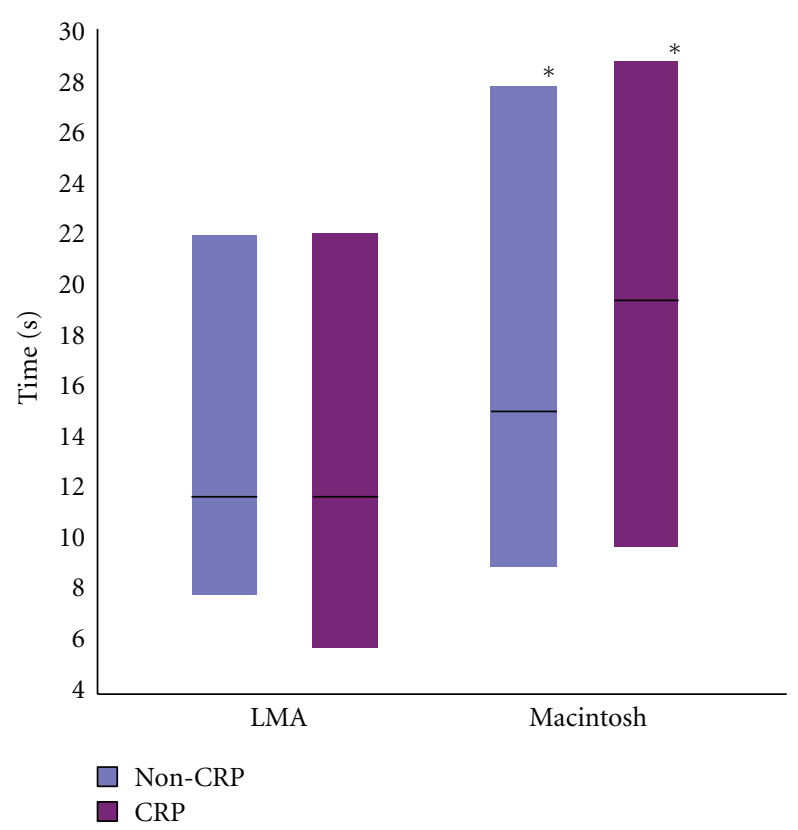

Figure 1: Time required for the first successful attempt for both techniques ${ }^{*} P<0.001$ LMA versus Macintosh blade; LMA: laryngeal mask airway. Block upper and lower values represent the range.

TABle 2: Participants' level of self-confidence in using the 2 devices; the values given are median with range.

\begin{tabular}{lcccc}
\hline \multirow{2}{*}{ Device } & \multicolumn{2}{c}{ LMA } & \multicolumn{2}{c}{ Macintosh blade } \\
& Non-CPR & CPR & Non-CPR & CPR \\
\hline Self-confidence & $8(3-10)$ & $8(2-9)$ & $6(3-8)$ & $4(2-7)$ \\
\hline
\end{tabular}

\section{Discussion}

Standard direct laryngoscopic tracheal intubation remains the preferred technique to establish advanced airway management, as correct placement of an endotracheal tube provides adequate tidal volume without stomach inflation [7]. However, endotracheal intubation is a technique that requires sufficient training and retraining, and its success is closely associated with the intubator's experience [1, 10, 11]. Furthermore, concern has been raised that the risks of failed intubation, misplaced tubes, and prolonged intubation times may outweigh the benefits [12-17]. In addition, direct laryngoscopy and tracheal intubation remain a challenge for medical practitioners who do not have clinical experience with the techniques or who are expected to perform intubation in difficult situations $[18,19]$. In this study, results showed that a percentage of newly trained paramedics failed to intubate the trachea, confirming the notion that intubation is a skill that is difficult to learn and maintain.

LMA was introduced into clinical practice almost 30 years ago, and it has replaced endotracheal intubation in many instances, such as in the Emergency Department or even in elective surgery [20]. It is widely known that supraglottic devices are easier to learn and use than endotracheal intubation $[2,3]$. In addition, inexperienced personnel are easier to be trained in using LMA than in intubating the trachea [21]. Unfortunately, it is also well known that in the clinical setting, many cardiac arrest victims experience vomiting of their gastric contents because their stomachs are often full and the mask-bag ventilations can cause regurgitation. The stomach contents sometimes lead to serious respiratory compromise by obstructing the upper airway, and the use of LMA does not protect against this. On the other hand, several animal studies have shown that frequent or prolonged interruption of chest compressions is associated with reduced coronary perfusion pressure, reduced return of spontaneous circulation, reduced survival rates, and reduced postresuscitation myocardial function [22-25]. This is the reason why the ERC guidelines stress that any placement of an advanced airway device should be achieved with the least possible interruption to chest compressions. Furthermore, time of securing the airway with any device is crucial and needs to be done as early and as quickly as possible. According to the results of this study, LMA insertion was more effective as all paramedics managed to place LMA and faster than endotracheal intubation. Furthermore, this study demonstrated that chest compressions prolonged tracheal intubation time and raised significantly the number of attempts, while LMA insertion times and number of attempts were not affected by chest compressions. These data support the use of LMA in resuscitation by paramedics not already experienced in intubation. Moreover, another interesting finding was that participants expressed a greater level of self-confidence in using the LMA instead of intubating the trachea with the Macintosh blade, a fact that should be taken in consideration when dealing with such a stressful situation as CPR setting. Finally, it is important to emphasize that uninterrupted chest compressions were performed in this study and according to our knowledge, such studies are very limited.

4.1. Limitations. This study has a number of significant limitations. Firstly, the study was performed in manikins and not in patients. Manikins never fully replicate real-time patient conditions and that is why it is difficult to predict how our findings would translate into clinical practice. Secondly, the investigators responsible for data acquisition were not blinded as to the technique used, as it would have been impossible to keep accurate track of time and not see the techniques assessed. Another limitation of the study was that we made no measure of the efficacy of ventilation in the manikin, requiring only that the chest rose visibly. We also made no measure of the extent of gastric inflation caused by each device.

In an attempt to improve outcomes in emergency situations, it is necessary for all healthcare personnel (medical or nonmedical) to acquire the skill of securing the airway with any of the available techniques. Due to the aforementioned disadvantages of endotracheal intubation, the use of LMA particularly for paramedics, nurses, and doctors who do not have regular exposure to situations in which airway management skills are required; in these situations, it is 
vital that an effective airway can be obtained quickly and without extensive prior training using the airway device. Time to ventilation and success rates should be considered as crucial criteria when evaluating airway management devices. Taking in consideration that paramedics are usually the first responders, our results provide evidence that LMA insertion is much faster and easier to handle than endotracheal intubation. Further studies towards this direction are required, before these can be implemented in every day clinical practice.

\section{Conflict of Interests}

The authors declared no conflict of interests.

\section{References}

[1] A. J. Hirsch-Allen, N. Ayas, S. Mountain, P. Dodek, A. Peets, and D. E. Griesdale, "Influence of residency training on multiple attempts at endotracheal intubation," Canadian Journal of Anaesthesia, vol. 57, no. 9, pp. 823-829, 2010.

[2] C. D. Deakin, R. Peters, P. Tomlinson, and M. Cassidy, "Securing the prehospital airway: a comparison of laryngeal mask insertion and endotracheal intubation by UK paramedics," Emergency Medicine Journal, vol. 22, no. 1, pp. 64-67, 2005.

[3] D. J. Reinhart and G. Simmons, "Comparison of placement of the laryngeal mask airway with endotracheal tube by paramedics and respiratory therapists," Annals of Emergency Medicine, vol. 24, no. 2, pp. 260-263, 1994.

[4] L. M. Jacobs, L. D. Berrizbeitia, B. Bennett, and C. Madigan, "Endotracheal intubation in the prehospital phase of emergency medical care," Journal of the American Medical Association, vol. 250, no. 16, pp. 2175-2177, 1983.

[5] A. J. Gray, D. Cartlidge, and M. C. Gavalas, "Can ambulance personnel intubate?" Archives of Emergency Medicine, vol. 9, no. 4, pp. 347-351, 1992.

[6] B. D. Shy, T. D. Rea, L. J. Becker, and M. S. Eisenberg, "Time to intubation and survival in prehospital cardiac arrest," Prehospital Emergency Care, vol. 8, no. 4, pp. 394-399, 2004.

[7] J. P. Nolan, C. D. Deakin, J. Soar, B. W. Böttiger, and G. Smith, "European Resuscitation Council guidelines for resuscitation 2005. Section 4. Adult advanced life support," Resuscitation, vol. 67, no. 1, pp. S39-S86, 2005.

[8] D. Yannopoulos, T. P. Aufderheide, A. Gabrielli et al., "Clinical and hemodynamic comparison of 15:2 and 30:2 compressionto- ventilation ratios for cardiopulmonary resuscitation," Critical Care Medicine, vol. 34, no. 5, pp. 1444-1449, 2006.

[9] K. Stroumpoulis, T. Xanthos, E. Bassiakou et al., "Macintosh and Glidescope performance by advanced cardiac life support providers: a manikin study," Minerva Anestesiologica, vol. 77, no. 1, pp. 11-16, 2011.

[10] M. J. Sagarin, E. D. Barton, Y. M. Chng, and R. M. Walls, "Airway management by US and Canadian emergency medicine residents: a multicenter analysis of more than 6,000 endotracheal intubation attempts," Annals of Emergency Medicine, vol. 46, no. 4, pp. 328-336, 2005.

[11] K. R. Stringer, S. Bajenov, and S. M. Yentis, "Training in airway management," Anaesthesia, vol. 57, no. 10, pp. 967-983, 2002.

[12] J. H. Jones, M. P. Murphy, R. L. Dickson, G. G. Somerville, and E. J. Brizendine, "Emergency physician-verified out-ofhospital intubation: miss rates by paramedics," Academic Emergency Medicine, vol. 11, no. 6, pp. 707-709, 2004.
[13] M. R. Sayre, J. C. Sakles, A. F. Mistler et al., "Field trial of endotracheal intubation by basic EMTs," Annals of Emergency Medicine, vol. 31, no. 2, pp. 228-233, 1998.

[14] J. D. Nolan, "Prehospital and resuscitative airway care: should the gold standard be reassessed?" Current Opinion in Critical Care, vol. 7, no. 6, pp. 413-421, 2001.

[15] H. E. Wang and D. M. Yealy, "Out-of-hospital endotracheal intubation: where are we?" Annals of Emergency Medicine, vol. 47, no. 6, pp. 532-541, 2006.

[16] S. H. Katz and J. L. Falk, "Misplaced endotracheal tubes by paramedics in an urban emergency medical services system," Annals of Emergency Medicine, vol. 37, no. 1, pp. 32-37, 2001.

[17] J. J. Gatward, M. J. Thomas, J. P. Nolan, and T. M. Cook, "Effect of chest compressions on the time taken to insert airway devices in a manikin," British Journal of Anaesthesia, vol. 100, no. 3, pp. 351-356, 2008.

[18] P. Gerbeaux, "Should emergency medical service rescuers be trained to practice endotracheal intubation?" Critical Care Medicine, vol. 33, no. 8, pp. 1864-1865, 2005.

[19] J. T. Mulcaster, J. Mills, O. R. Hung et al., "Laryngoscopic intubation: learning and performance," Anesthesiology, vol. 98, no. 1, pp. 23-27, 2003.

[20] A. I. Brain, "The laryngeal mask: a new concept in airway management," British Journal of Anaesthesia, vol. 55, no. 8, pp. 801-805, 1983.

[21] B. M. Wahlen, N. Roewer, M. Lange, and P. Kranke, “Tracheal intubation and alternative airway management devices used by healthcare professionals with different level of pre-existing skills: a manikin study," Anaesthesia, vol. 64, no. 5, pp. 549554, 2009.

[22] R. A. Berg, L. A. Cobb, A. Doherty et al., "Chest compressions and basic life support-defibrillation," Annals of Emergency Medicine, vol. 37, no. 4, pp. S26-S35, 2001.

[23] K. B. Kern, R. W. Hilwig, R. A. Berg, A. B. Sanders, and G. A. Ewy, "Importance of continuous chest compressions during cardiopulmonary resuscitation: improved outcome during a simulated single lay-rescuer scenario," Circulation, vol. 105, no. 5, pp. 645-649, 2002.

[24] T. Yu, M. H. Weil, W. Tang et al., "Adverse outcomes of interrupted precordial compression during automated defibrillation," Circulation, vol. 106, no. 3, pp. 368-372, 2002.

[25] R. A. Berg, R. W. Hilwig, K. B. Kern, A. B. Sanders, L. C. Xavier, and G. A. Ewy, "Automated external defibrillation versus manual defibrillation for prolonged ventricular fibrillation: lethal delays of chest compressions before and after countershocks," Annals of Emergency Medicine, vol. 42, no. 4, pp. 458-467, 2003. 


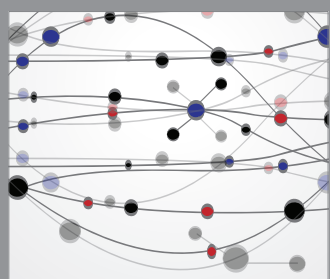

The Scientific World Journal
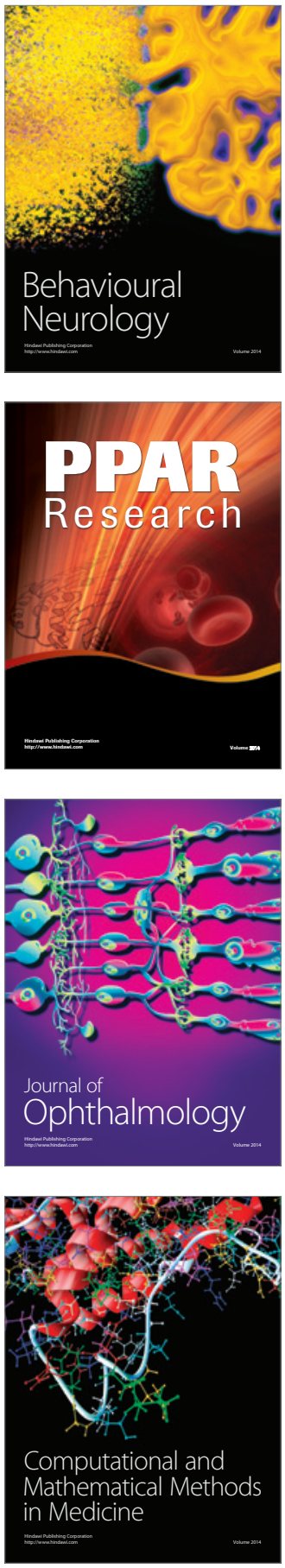

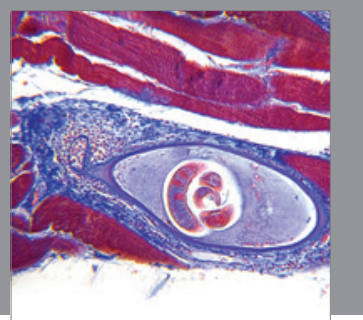

Gastroenterology

Research and Practice
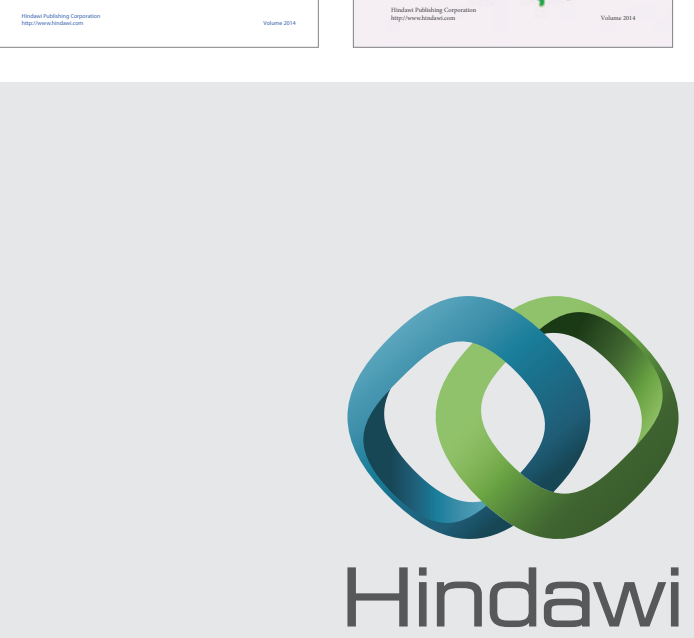

Submit your manuscripts at

http://www.hindawi.com
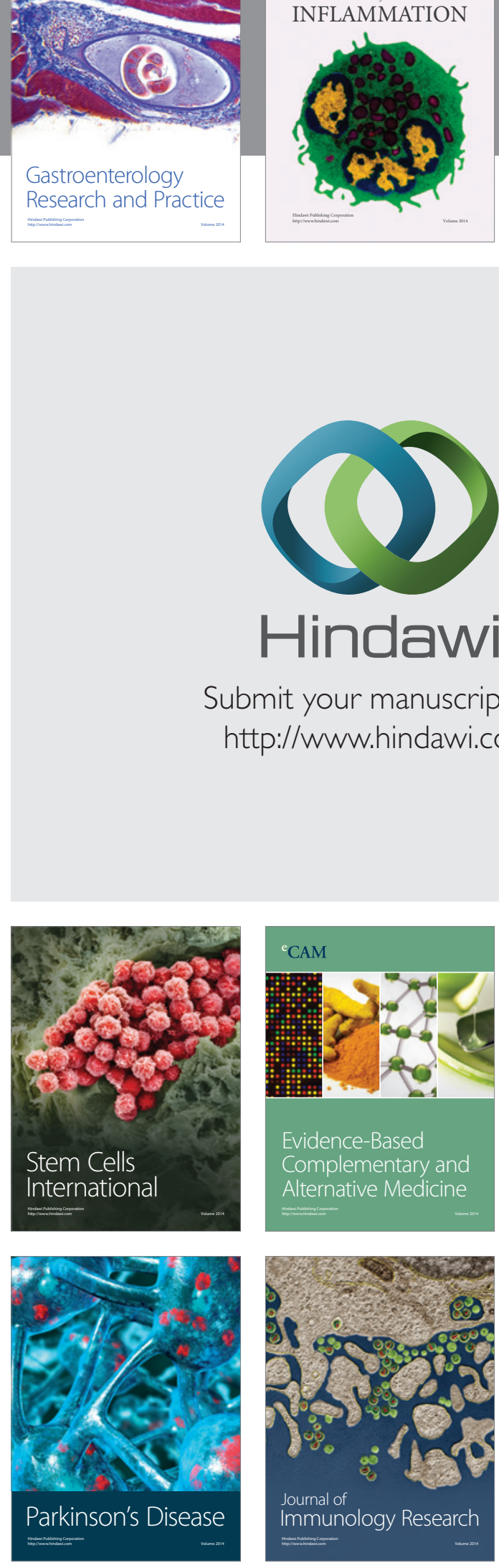

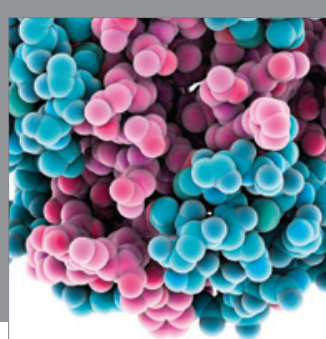

Diabetes Research
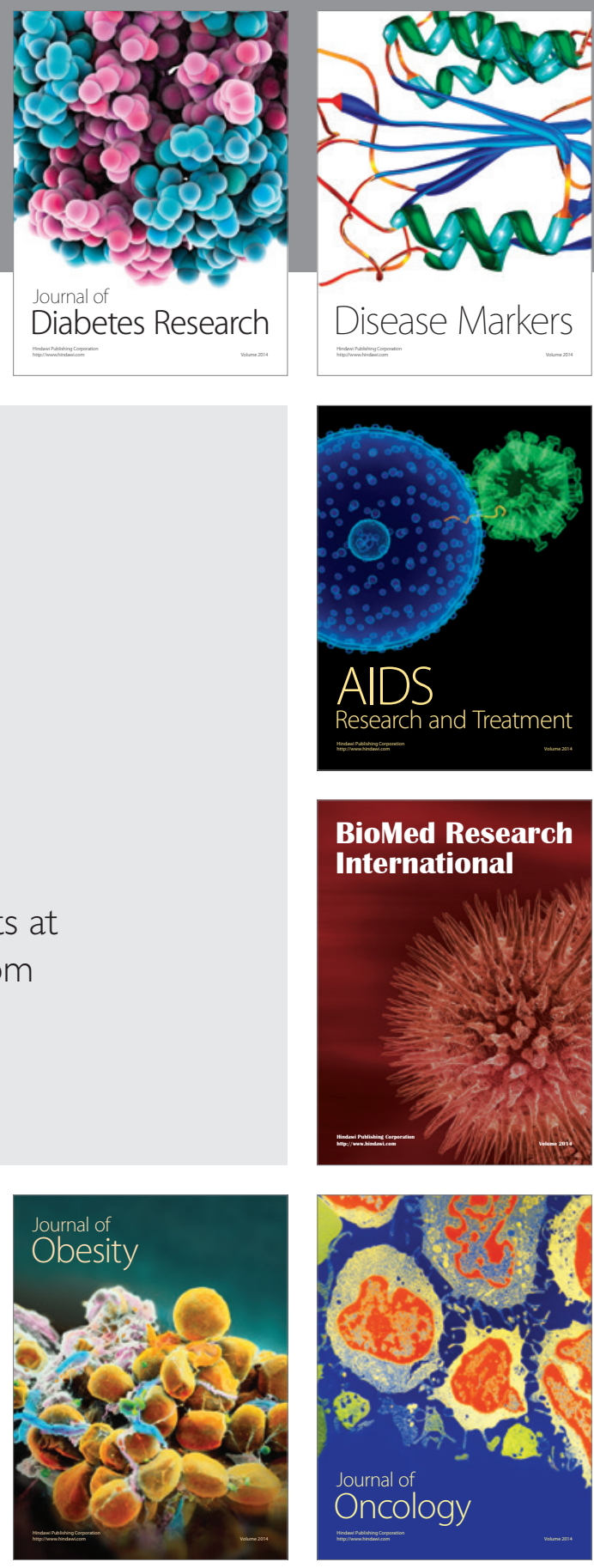

Disease Markers

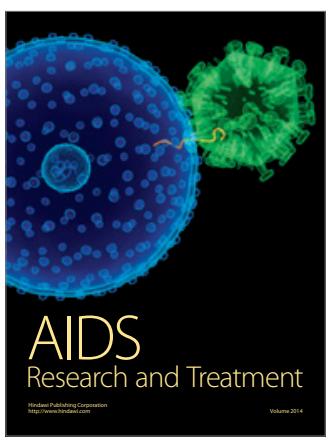

BioMed Research

International
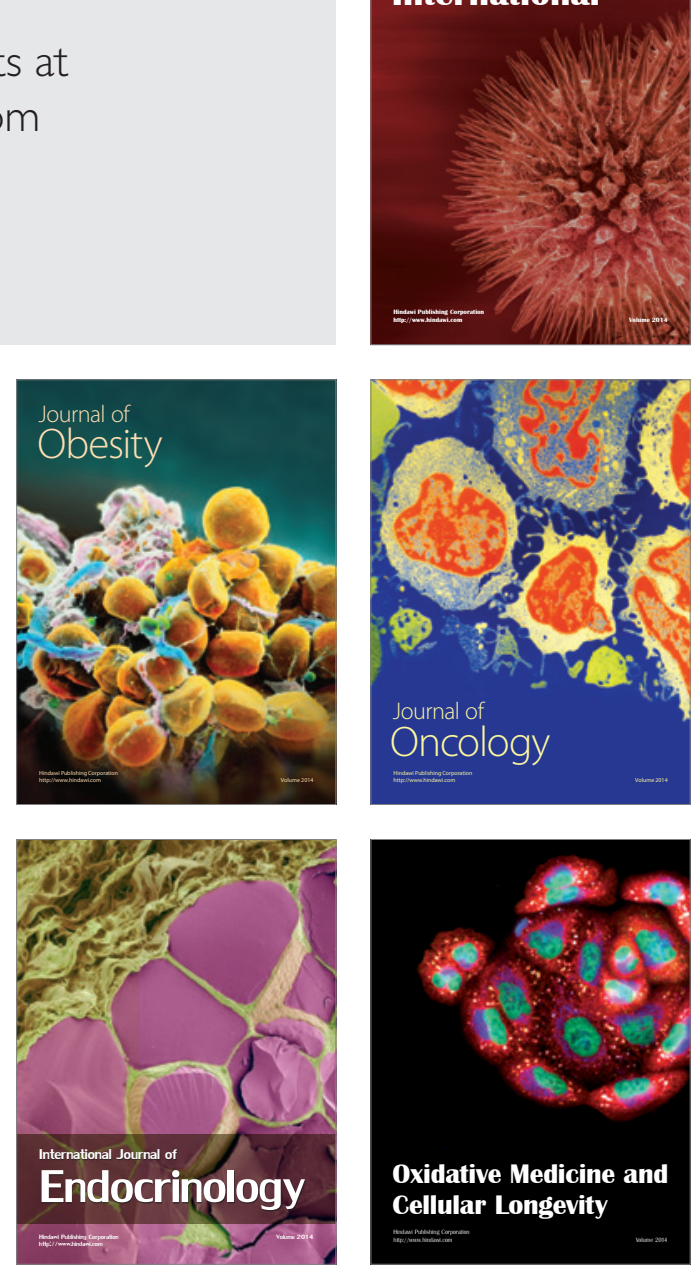